\title{
Similarity thresholds used in DNA sequence assembly from short reads can reduce the comparability of population histories across species
}

Michael G Harvey, Caroline Duffie Judy, Glenn F Seeholzer, James M Maley, Gary R Graves, Robb T Brumfield

Comparing inferences among datasets generated using short read sequencing may provide insight into the concerted impacts of divergence, gene flow and selection across organisms, but comparisons are complicated by biases introduced during dataset assembly. Sequence similarity thresholds allow thede novoassembly of short reads into clusters of alleles representing different loci, but the resulting datasets are sensitive to both the similarity threshold used and to the variation naturally present in the organism under study. Stringent thresholds, those requiring high sequence similarity among reads for assembly, as well as highly variable species may result in datasets in which divergent alleles are lost or divided into separate loci ('over-splitting'), whereas liberal thresholds increase the risk of paralogous loci being combined into a single locus ('under-splitting'). Comparisons among datasets or species are therefore potentially biased if different similarity thresholds are applied or if the species differ in levels of within-lineage genetic variation. We examine the impact of a range of similarity thresholds on assembly of empirical short read datasets from populations of four different non-model bird lineages (species or species pairs) with different levels of genetic divergence. We find that, in all species, stringent similarity thresholds result in fewer alleles per locus than more liberal thresholds, which appears to be the result of high levels of over-splitting. The frequency of putative under-splitting, conversely, is low at all thresholds. Inferred genetic distances between individuals, gene tree depths, and estimates of the ancestral mutation-scaled effective population size $(\theta)$ differ depending upon the similarity threshold applied. Relative differences in inferences across species differ even when the same threshold is applied, but may be dramatically different when datasets assembled under different thresholds are compared. These differences not only complicate comparisons across species, but also preclude the application of standard mutation rates for parameter calibration. We suggest some best practices for assembling short read data to maximize comparability, such as using more liberal thresholds and examining the impact of different thresholds on each dataset. 
Museum of Natural Science, Louisiana State University, Baton Rouge, LA 70803, USA

Department of Biological Sciences, Louisiana State University, Baton Rouge, LA 70803, USA

James M. Maley

Museum of Natural Science, Louisiana State University, Baton Rouge, LA 70803, USA Department of Biological Sciences, Louisiana State University, Baton Rouge, LA 70803, USA Moore Laboratory of Zoology, Occidental College, Los Angeles, CA 90041 USA

Gary R. Graves

Department of Vertebrate Zoology, MRC-116, National Museum of Natural History, Smithsonian Institution, Washington, DC 20013, USA

Center for Macroecology, Evolution and Climate, Natural History Museum of Denmark,University of Copenhagen, DK-2100, Copenhagen Ø, Denmark

\section{Robb T. Brumfield}

Museum of Natural Science, Louisiana State University, Baton Rouge, LA 70803, USA

Department of Biological Sciences, Louisiana State University, Baton Rouge, LA 70803, USA

Corresponding author:

Michael G. Harvey

Museum of Natural Science

119 Foster Hall

Louisiana State University

Baton Rouge, LA 70803, USA

225-578-2855

mharve9@1su.edu 
INTRODUCTION

With the proliferation of population-level datasets obtained using massively parallel sequencing technologies, there is increasing interest in studies that compare inferences from genomic datasets

51 obtained from different species (e.g., Leaché et al., 2013; Smith et al., 2013) or from different genomic

52 regions (e.g., Evans et al., 2014; Harvey et al., 2013; Leaché et al., 2015). Assembly of short sequence

53 reads into orthologous loci is a key component of post-sequence processing, and commonly used methods can lead to biases in population genetic parameter estimation (Ilut, Nydam \& Hare, 2014). Here, we explore the effect of one major source of bias on the comparability of datasets and inferences.

Sequence similarity provides the information necessary for assembling reads into orthologous loci (Pop \& Salzberg, 2008; Chaisson, Brinza \& Pevzner, 2009). By setting a sequence similarity threshold, researchers attempt to assemble similar, presumably orthologous reads into loci while separating or removing dissimilar, presumably non-orthologous reads (e.g. Etter et al., 2011; Catchen et al., 2011). Selecting the most appropriate similarity threshold is challenging, primarily because the

61 amount of genetic (allelic) variation can vary greatly among orthologous loci within a species (Ilut,

62 Nydam \& Hare, 2014). Because the amount of genetic variation also varies among species and

63 genomic regions, a particular similarity threshold may impact each dataset differently, potentially

64 influencing inferences in comparative studies. Many methods default to a stringent similarity threshold, often requiring 98-99\% sequence

66 similarity among reads for assembly (e.g., Catchen et al., 2011; Lu et al., 2013). However, stringent

67 similarity thresholds may split orthologous reads into multiple loci if the reads come from alleles that 68 are more different than the threshold permits (hereafter "over-splitting"; Fig. 1a). More liberal

69 similarity thresholds permit the assembly of more dissimilar orthologous reads into loci, but are more 70 susceptible to including paralogous reads in the assembly (hereafter "under-splitting"; Fig. 1b). Using 
71 simulations, Rubin, Ree and Moreau (2012) found that under-splitting was frequent at more liberal

72 similarity thresholds in phylogenetic datasets, but did not strongly bias inference. Catchen et al. (2013)

73 examined RAD-Seq data from three-spined sticklebacks, and found that over-splitting was an issue

74 when datasets were processed with similarity thresholds more stringent than $96 \%$. Ilut, Nydam and

75 Hare (2014) tested the impact of similarity threshold selection on both over- and under-splitting in

76 three simulated and one empirical RAD-Seq dataset. They found that under-splitting was minimal and

77 that affected loci were easily identified due to the presence of individuals with more alleles than

78 expected given their ploidy, but that over-splitting was significant at more stringent similarity

79 thresholds.

Comparative phylogeographic and population genetics studies are particularly susceptible to

81 biases resulting from similarity thresholds, particularly over-splitting. Different species often exhibit

82 different levels of genetic diversity (Lewontin, 1974; Taberlet et al., 1998; Smith et al., 2014;

83 Romiguier et al., 2014), and this variation across species may interact with the application of similarity

84 thresholds to differentially bias datasets. Huang and Knowles (In press), for example, found that

85 mutational spectra of datasets simulated under deeper species trees were biased downwardly relative to

86 those simulated under shallow species trees when processed with the same similarity threshold (both

$8798 \%$ and $95 \%$ similarity were examined). The effects of similarity thresholds have not been examined,

88 however, using empirical data from species that vary in their levels of genetic diversity. Although

89 diverse parameters required for short read assembly are worthy of scrutiny, we focus on similarity

90 thresholds as they are particularly important for maintaining comparability across species with

91 different levels of variation.

In this study, we examine the effect of similarity thresholds on dataset assembly and

93 phylogeographic inferences across four non-model bird lineages that vary in divergence. We sample

94 two populations or species within each lineage and assemble a RAD-Seq dataset for each species at a 
95 series of similarity thresholds to assess the impact of different thresholds on the number of unique

96 alleles observed within assembled loci. We investigate the effect of different similarity thresholds on

97 estimates of standard population genetic and phylogeographic parameters within species and in

98 comparisons across species.

99

100

101 MATERIALS AND METHODS

102

103 Study Species and Sampling

104

105

We sampled four individuals from each of two populations, as determined based on taxonomy

106 and prior genetic data, in four lineages (Table S1). The first lineage includes Clapper (Rallus crepitans

107 J. F. Gmelin, 1788) and King (R. elegans J. J. Audubon, 1834) rails, sister species of medium-sized

108 water birds that interbreed in a narrow hybrid zone centered on a salinity gradient (Maley, 2012; Maley

$109 \&$ Brumfield, 2013). We also examined the Streamertail (Trochilus polytmus C. Linnaeus, 1758), a

110 hummingbird endemic to Jamaica that comprises two subspecies (T. p. polytmus and T. p. scitulus) that

111 differ primarily in bill coloration and interbreed in a narrow hybrid zone (Gill et al., 1973; Coyne \&

112 Price, 2000). Line-cheeked (Cranioleuca antisiensis P. L. Sclater, 1859) and Baron's (Cranioleuca

113 baroni O. Salvin, 1895) Spinetail are closely related, small insectivorous birds distributed along the

114 Andes Mountains (Remsen, 2003). Finally, we sampled two populations of Plain Xenops (Xenops

115 minutus A. E. Sparrman, 1788), a widespread insectivorous bird of lowland Neotropical forests that are

116 separated by the Andes and differ in plumage, voice, and genetic markers (Remsen, 2003; Burney,

117 2009; Harvey \& Brumfield, 2015).

118 
For each individual examined, we extracted total DNA from vouchered tissue samples using

DNeasy tissue kits (Qiagen, Valencia, CA, USA) following the manufacturer's protocol. We sent DNA

123 extracts to the Cornell Institute of Genomic Diversity (IGD) to collect data using Genotyping by

124 Sequencing, a RAD-Seq method (Elshire et al., 2011). Briefly, the IGD digested DNA using PstI

125 (CTGCAG) and ligated a sample-specific indexed adapter and common adapter to resulting fragments.

126 The IGD pooled and cleaned ligated samples using a QIAquick PCR purification kit (Qiagen),

127 amplified the pool using an 18-cycle PCR, purified the PCR product using QIAquick columns, and

128 quantified the amplified libraries using a PicoGreen assay (Molecular Probes, Carlsbad, CA, USA).

129 Based on the PicoGreen concentrations, the IGD then combined the samples for this project with

130 unrelated samples and ran plates of 96 samples on a 100-base pair, single-end Illumina HiSeq 2000

131 lane.

133 Bioinformatics Processing

We processed the raw GBS reads using the Stacks pipeline (Catchen et al., 2011; 2013) due to

136 its popularity in prior studies assembling RAD-Seq datasets within species. Although other dataset

137 assembly programs are available (e.g. Eaton, 2014; Sovic et al., in press), all rely on similarity

138 thresholds and should yield similar results with respect to the analyses presented here. Datasets were

139 assembled on compute nodes (2.93 GHz Quad Core Nehalem Xeon 64-bt processors with $24 \mathrm{~GB} 1333$

$140 \mathrm{MHz}$ RAM or 96GB 1066MHz RAM) maintained by LSU High Performance Computing. We

141 demultiplexed raw reads, cleaned reads, and removed barcode and adapter sequences using the

142 program process_radtags.pl. We assembled alleles and loci de novo using the program denovo_map.pl. 
143 We used custom Python (Python Software Foundation, 2007) scripts (available at

144 https:/github.com/mgharvey/misc_Python) to obtain sequence alignments of both alleles for each

145 individual from the Stacks output files. Detailed settings are provided in the supplement (Table S2).

146 To investigate the impact of similarity thresholds on dataset attributes and downstream

147 analyses, we assembled seven datasets for each of the four lineages under similarity thresholds (Stacks

148 settings $-\mathrm{M}$ and $-\mathrm{n}$ ) at all integer values from 93\% (7 mismatches allowed) to 99\% (1 mismatch

149 allowed), reflecting the range of settings typically used for assembling intraspecific datasets. Assembly

150 with similarity thresholds less stringent than $93 \%$ failed due to high computational demand in Stacks,

151 but should not be necessary for the divergences examined here or for most other population-level

152 studies. Reads with similarity values above the selected threshold clustered into assemblies, which we

153 treated as independently segregating loci in downstream analyses. We disabled the use of secondary,

154 more divergent reads for calling genotypes (Stacks setting $-\mathrm{H}$ ) to prevent the assembly of reads that are

155 less similar than the similarity threshold used for primary stacks. We set minimum depth per allele

156 (Stacks setting -m) to ten, which provides a balance between the inclusion of singleton alleles

157 (potential errors) and the total size of the data matrix (Fig. S1). We set the maximum number of alleles

158 per individual (Stacks setting --max_locus_stacks) to three, one above the ploidy level of the study

159 organisms. In the resulting datasets, this setting will result in three called alleles for any individuals

160 containing three or more alleles, allowing the identification of alignments containing reads from

161 paralogous loci. We used custom Python scripts to format files and calculate basic statistics and used

162 COMPUTE (Thornton, 2003) to estimate standard population genetic summary statistics.

163 Monomorphic loci as well as those with variable sites were retained in all subsequent analyses unless

164 otherwise specified. 
168 We examined the number of unique alleles per locus across treatments to examine how

169 different similarity thresholds affected each dataset. As an index of the frequency of under-splitting in

170 each dataset, we calculated the number of loci containing individuals with more than two alleles. These

171 loci were presumed to contain paralogous reads and were removed from further analysis. To assess the

172 proportion of loci with putative over-split alleles, we mapped loci assembled under the more stringent

173 thresholds (94-99\%) to the set of loci assembled under the most liberal threshold (93\%). This allowed

174 us to detect instances in which multiple loci from the more stringent threshold mapped to the same

175 locus from the liberal threshold. We used lastz (Harris, 2007) for mapping with minimum identity set

176 at $93 \%$ for all comparisons and no gaps permitted. We subtracted from each total the number of loci

177 from the liberal threshold (93\%) that mapped to other loci assembled with the same threshold using

178 lastz.

179

180

Genetic Distances and $F_{s t}$

181

182

Over-splitting may reduce estimates of genetic distance between individuals or populations if

183 they contain dissimilar alleles. Conversely, if over-splitting reduces the number of alleles within

184 populations, this may reduce estimates of distance between populations. We calculated pairwise p-

185 distances and Jukes-Cantor corrected distances per unit sequence length at each locus. We measured

186 distances between individuals by measuring the average distance between alleles in the first individual

187 and those in the second individual. For loci containing variable sites, we also estimated $\mathrm{F}_{\mathrm{st}}$ between the

188 two populations within each lineage using formula (3) of Hudson, Slatkin and Maddison (1992), which

189 is based on the ratio of the mean number of differences between different sequences sampled within

190 populations to the mean number of differences between sequences sampled between populations. 
Gene Trees

193

Over-splitting may also reduce average gene tree depth due to the loss of more variable loci

195 owing to them being subdivided into two or more less variable loci. To reduce computation, we

196 selected a random subsample of 1,000 loci for each lineage at each threshold for gene tree estimation.

197 We selected the best-fit finite sites substitution model for each locus using mrAIC.pl (Nylander, 2004)

198 and conducted MrBayes (Ronquist and Huelsenbeck, 2003) runs with a random starting tree, four

199 Markov chains, and a 100,000-iteration burn-in followed by 1,000,000 sampling iterations. We

200 measured the depth of gene trees as mean depth of the deepest node in number of expected

201 substitutions using the R (R Core Team, 2014) package ape (Paradis, Claude \& Strimmer, 2004).

Demographic Parameter Estimation

We used the 1,000 locus subsets from gene tree estimation to estimate ancestral and contemporary population sizes in each lineage at each similarity threshold using the coalescent model implemented in BP\&P (Yang \& Rannala, 2010). Although this method assumes no gene flow between populations, which may be violated in some of our study lineages, simulations have demonstrated that BP\&P performance is robust to limited gene flow (Zhang et al., 2011). We used a speciation model

210 containing two contemporary populations and a divergence time parameter $(\tau)$ as well as population

211 standardized mutation rate parameters $\left(\theta=4 N_{e} \mu\right.$, where $N_{e}$ is the effective population size and $\mu$ is the 212 substitution rate per site per generation) for both daughter populations and an ancestral population. We 213 set prior values using gamma distributions determined by a shape parameter $(\alpha)$ and scale parameter 
214 ( $\beta$ ). Priors for both divergence time and population standardized mutation rate were set to $\alpha=1$ and $\beta$ $215=300$. We ran analyses for a burn-in of 50,000 iterations and then sampled every other iteration for an 216 additional 500,000 iterations.

RESULTS

After removing loci containing putative paralogous reads (see below), we recovered between

96,776 and 158,328 loci for the four lineages across the range of similarity thresholds examined (Table

1). The similarity threshold used had an effect on the number of unique alleles per locus in all four

224 lineages (Kruskal Wallis test $\mathrm{p}<2.20^{-16}$; Table S3). The number of alleles was low using the $99 \%$ 225 similarity threshold, but increased and plateaued as the threshold approached 93\% (Fig. 2a). The 226 number of alleles was more similar across lineages at stringent thresholds relative to liberal thresholds.

227 For example, Xenops contained, on average, 1.4 times as many alleles as Rallus when processed with a $22899 \%$ similarity threshold, but 1.66 times as many alleles when processed with a $93 \%$ similarity 229 threshold.

230 The proportion of loci containing putative paralogous reads (under-split loci) increased slightly 231 with increasing similarity thresholds, but was less than $0.4 \%$ at all thresholds for all lineages (Fig. 2b). 232 At all thresholds, Trochilus exhibited roughly half the level of putative paralogy displayed in the other 233 lineages (Table S4). Depending on the lineage, $5-61 \%$ of loci represented putative over-split alleles 234 based on lastz mapping at the most stringent similarity threshold of $99 \%$, but putative over-split alleles 235 decreased as thresholds became more liberal (Fig. 2b). 
Both uncorrected $\mathrm{p}$ and Jukes-Cantor corrected genetic distances between individuals were

237 reduced at more stringent similarity thresholds (Fig. 3a). Variance across lineages in mean genetic

238 distance increased as similarity thresholds became more liberal (Fig. S2), although relative values

239 between lineages were similar across thresholds. $\mathrm{F}_{\text {st }}$ estimates between populations did not differ across

240 thresholds (Fig. 3b).

241 Mean gene tree depth, based on the depth of the deepest node, increased as more liberal

242 similarity thresholds were applied in each lineage (Fig. 3c). Variance in mean gene tree depths across

243 lineages was inversely related to threshold stringency (Fig. S2) and relative values across lineages were

244 contingent on the threshold applied. For example, the mean gene tree depth for Xenops was $1.48 \times$

245 greater than for Rallus at $99 \%$ similarity, but $1.91 \times$ greater at $93 \%$ similarity.

246 Ancestral $\theta$ estimates were higher at more liberal similarity thresholds for all four lineages (Fig

$2473 \mathrm{~d}$ ), but contemporary $\theta$ estimates and population divergence times $(\tau)$ showed no association with

248 similarity thresholds (Figs. S3, S4). Ancestral $\theta$ estimates, as with genetic distance and gene tree depth,

249 displayed lower variance across lineages at stringent relative to liberal thresholds (Fig. S2). Relative

250 values across lineages also differed across thresholds. The ancestral $\theta$ for Xenops was $1.89 \times$ greater

251 than for Rallus at $99 \%$ similarity, for example, but $2.95 \times$ greater at $93 \%$ similarity.

DISCUSSION

Comparability of parameter estimates is essential for comparative studies of phylogeographic 257 structure and genetic diversity across species or among genomic regions (Nybom, 2004). Our results 258 reveal, however, that inferences differ not only among lineages with different population histories, but 
259 also according to the similarity threshold applied during dataset assembly. Differences in the impact of

260 similarity thresholds across datasets not only reduce the utility of those datasets for comparative

261 studies, but also preclude the application of standardized mutation rate estimates that would allow

262 demographic parameters in non-model species to be converted to real values (DaCosta \& Sorenson,

263 2014). The issues discussed here are not restricted to RAD-Seq datasets, but are of concern for all short

264 read datasets requiring similarity-based de novo assembly, including those from sequence capture and

265 transcriptomic approaches. Mapping reads to existing reference sequences also requires the application

266 of similarity thresholds and, although identifying under-splitting is more straightforward with a

267 reference genome, divergent alleles may still be lost if the threshold used for mapping is too stringent

268 (Trapnell \& Salzberg, 2009; Lunter \& Goodson, 2011). In such cases, over-splitting results in the loss

269 of alleles divergent from the reference, rather than the splitting of alleles into separate loci. Careful

270 selection of similarity thresholds for assembly is an important issue for diverse sequencing projects,

271 particularly if comparisons are to be made across datasets.

272

We found that datasets assembled under stringent similarity thresholds included fewer unique

273 alleles per locus than those assembled under more liberal thresholds. Similarly, Ilut, Nydam and Hare

274 (2014) found heterozygosity was reduced when stringent similarity thresholds were applied, but

275 increased with more liberal thresholds across three simulated and one empirical dataset. The reduced

276 number of alleles per locus in datasets assembled with stringent thresholds is likely due to the higher

277 frequency of putative over-splitting in those datasets. Prior studies also demonstrated that over-splitting

278 is frequent when datasets are processed at stringent similarity thresholds, and that this leads to allele

279 loss (Catchen et al., 2013; Ilut, Nydam \& Hare, 2014). Our results suggest that under-splitting occurs at

280 low frequencies across similarity thresholds and has little impact on datasets. The impact of under-

281 splitting may be more severe in species with highly repetitive genomes or in studies across deep, 
282 phylogenetic timescales that require more liberal similarity thresholds for assembly (e.g., Rubin, Ree \& 283 Moreau, 2012; Eaton \& Ree, 2013).

Variation in datasets resulting from the similarity threshold applied has important effects on downstream parameter estimation. In addition to the biases in population genetic and phylogeographic estimates that we found, Huang and Knowles (In press) found that mutational spectra are downwardbiased as a result of the loss of the most divergent alleles, and some studies have found that simulated data (Rubin, Ree \& Moreau, 2012; Huang \& Knowles, in press). Unlike other parameters, our $\mathrm{F}_{\mathrm{st}}$ estimates were not strongly impacted by variation in similarity thresholds, perhaps because $\mathrm{F}_{\mathrm{st}}$ is

291 calculated using the ratio of between- and within-population divergence, both of which are impacted by allele loss. In addition, $\theta$ values from contemporary populations were similar across thresholds, while ancestral $\theta$ values were lower at more stringent thresholds. This may result if stringent thresholds result

294 in the loss of alleles that are fixed between the two divergent populations at a higher rate than those 295 that are variable within populations. Despite these exceptions, it seems likely that observed biases in 296 datasets across similarity thresholds would impact diverse population genetic and phylogeographic 297 parameter estimates.

299 studies (e.g. Emerson et al., 2010; Reitzel et al., 2013; Chu et al., 2014), perhaps under the supposition 300 that they are more conservative and less likely to permit the assembly of non-orthologous reads or as 301 an attempt to reduce dataset size and computation times (Ilut, Nydam \& Hare, 2014). We concur with 302 Ilut, Nydam and Hare (2014) and Huang and Knowles (In press) that defaulting to stringent thresholds 303 is generally not appropriate. Over-splitting decreases at more liberal similarity thresholds and the 304 number of alleles per locus asymptotes near the $96 \%$ threshold, suggesting that datasets assembled 
305 under similarity thresholds of $96 \%$ or less stringency are relatively less biased by over-splitting.

306 Although this asymptote will vary depending on the divergence within a dataset, other studies have

307 found asymptotes at similar threshold values, for example at roughly $95-96 \%$ in empirical data from

308 sticklebacks (Catchen et al., 2013) or between roughly $88 \%$ and $96 \%$ in simulated tunicate,

309 stickleback, and soybean datasets and an empirical tunicate dataset (Ilut, Nydam \& Hare, 2014). The

310 approach suggested by Ilut, Nydam and Hare (2014) in which datasets are assembled at a series of

311 similarity thresholds, the location of the asymptote in over-splitting is identified, and that threshold is

312 used for final assembly is preferable to defaulting to stringent thresholds.

313 We were unable to directly investigate the frequency of under-splitting and over-splitting in our

314 datasets because we lack genome sequences for the non-model organisms examined. Our indirect

315 measure of over-splitting may detect not just over-split loci, but also loci that are under-split in the

316 assembly from the most liberal threshold but correctly separated in the assembly from the more

317 stringent thresholds. This would be particularly likely if paralogy was common in the genomes under

318 investigation or if very liberal similarity thresholds were examined. The frequency of under-splitting

319 appears to be low enough in our datasets, however, that this effect would be minimal. Broad

320 concordance between our results and prior investigations into over-splitting in systems with a genome

321 for reference (Catchen et al., 2013; Ilut, Nydam \& Hare, 2014) suggest that our metric of over-split

322 alleles is a reasonable proxy for use in non-model organisms.

323 Results from our indirect measure of under-splitting are also broadly consistent with the low

324 levels of under-splitting observed in prior work using reference genomes (Ilut, Nydam \& Hare, 2014)

325 and were expected given the low level of paralogy in avian genomes (e.g. chicken; Hillier et al., 2004).

326 Our measure of under-splitting, the number of loci containing individuals with more alleles than

327 expected, has been used previously to filter out loci with paralogous data from RAD-Seq datasets

328 (Parchman et al., 2012; Peterson et al., 2012). Some loci may contain reads from paralogous loci but 
may not contain sufficient numbers of alleles to trip this filter, potentially inflating estimates of variation. Prior work, however, suggests that paralogous reads lack strong signal conflicting with that from entirely orthologous loci and have relatively minor effects on inferences (Rubin, Ree \& Moreau, 2011). Other indicators such as extreme heterozygosity or other deviations from Hardy-Weinberg or linkage equilibrium in presumed panmictic populations (Catchen et al., 2011; White et al., 2013), violations of Mendelian inheritance in pedigreed individuals, or gene tree topologies suggesting a history of duplication might also be used to detect additional loci containing paralogous reads. These metrics deserve consideration in situations where under-splitting is a concern, including in species with high levels of heterozygosity or deep divergences (e.g., phylogenetic studies) necessitating the application of very liberal similarity thresholds or in species with highly repetitive genomes.

We uncovered differences in allelic diversity and parameter estimates across the four study lineages examined. Xenops minutus generally displayed the greatest allelic diversity and also the largest genetic distances between individuals, deepest gene trees, and highest $\theta$ values, which was perhaps not surprising given prior evidence of deep genetic divergences within this species (Smith et al., 2014; Harvey \& Brumfield, 2015). The other lineages were more similar by most measures, although Trochilus polytmus was slightly higher than Cranioleuca and Rallus in allelic diversity, genetic distance, and gene tree depths. Interestingly, Trochilus polytmus also exhibited roughly half the frequency of putative paralogous loci of the other three species, which may be related to the small genome size of hummingbirds (Gregory et al., 2009).

Our results suggest that the similarity threshold used for assembly impacts the level of variation in a dataset as well as downstream population genetic and phylogeographic estimates. Comparisons across datasets are also biased by the impact of similarity thresholds, appearing more similar across datasets when stringent thresholds are used or in some cases more different if species are assembled 
352 with different thresholds. These biases further preclude the estimation of standardized mutation rates

353 for parameter calibration. Methods for threshold selection exist that limit these biases, such as the use

354 of liberal thresholds and examination of the impact of a range of thresholds on a given dataset, but they

355 need to be further developed and applied more widely if we are to be able to compare datasets and

356 integrate inferences across studies, genomic regions, and organisms.

357

358

359

360

361 We thank the many collectors and museum curators and staff involved in obtaining and maintaining the 362 samples used for this study, in particular John M. Bates and David E. Willard (FMNH), Mark B.

363 Robbins (KUMNH), Donna L. Dittmann (LSUMNS), and James Dean (USNM). LSU High

364 Performance Computing provided computer resources. Jeremy M. Brown, Michael E. Hellberg,

365 Prosanta Chakrabarty, Jacob A. Esselstyn, and the LSU Vert Lunch group provided helpful comments

366 on the issue addressed in this paper. We thank Scott V. Edwards, Ryan C. Garrick, Magnus Popp, Paul

367 Hohonlohe, and three anonymous reviewers for constructive comments that improved the manuscript. 368 369
Burney CW. 2009. Comparative phylogeography of Neotropical birds. D. Phil. Dissertation, Louisiana State University. 
375 Catchen JM, Amores A, Hohenlohe PA, Cresko WA, Postlethwait JH. 2011. Stacks: building and genotyping loci de novo from short-read sequences. G3 Genes Genomes Genetics 1:171-182.

378 Catchen JM, Hohenlohe PA, Bassham S, Amores A, Cresko WA. 2013. Stacks: an analysis tool set for population genomics. Molecular Ecology 22:3124-3140.

381 Chaisson MJ, Brinza D, Pevzner PA. 2009. De novo fragment assembly with short mate-paired reads: does the read length matter? Genome Research 19:336-346.

384 Chu ND, Kaluziak ST, Trussell GC, Vollmer SV. 2014. Phylogenomic analyses reveal latitudinal 385 population structure and polymorphisms in heat stress genes in the North Atlantic snail Nucella lapillus. Molecular Ecology 23:1863-1873.

391 Eaton DA. 2014. PyRAD: Assembly of de novo RADseq loci for phylogenetic analyses. Bioinformatics 30:1844-1849.

394 Eaton DAR, Ree RH. 2013. Inferring phylogeny and introgression using RADseq data: An example 395 from flowering plants (Pedicularis: Orobanchaceae). Systematic Biology 62:689-706. 
397 Elshire RJ, Glaubitz JC, Sun Q, Poland JA, Kawamoto K, Buckler ES, Mitchell SE. 2011. A robust, 398 simple genotyping-by-sequencing (GBS) approach for high diversity species. PloS One 6:e19379.

400

401 Emerson KJ, Merz CR, Catchen JM, Hohenlohe PA, Cresko WA, Bradshaw WE, Holzapfel CM. 2010.

402 Resolving postglacial phylogeography using high-throughput sequencing. Proceedings of the National Academy of Sciences 107:16196-16200.

Etter PD, Preston JL, Bassham S, Cresko WA, Johnson EA. 2011. Local de novo assembly of RAD paired-end contigs using short sequencing reads. PLoS One 6:e18561.

407

408 409 sequencing suggests low diversity on the sex chromosomes of Tonkean macaque monkeys. Molecular Biology and Evolution 31:2425-2440.

Gill F, Stokes C. 1973. Contact zones and hybridization in the Jamaican hummingbird, Trochilus polytmus (L.). Condor 75:170-176.

Gregory TR, Andrews CB, McGuire JA, Witt CC. 2009. The smallest avian genomes are found in hummingbirds. Proceedings of the Royal Society B 276:3753-3757.

Harris RS. 2007. Improved pairwise alignment of genomic DNA. D. Phil. Dissertation, The Pennsylvania State University. 
421 Harvey MG, Brumfield RT. 2015. Genomic variation in a widespread Neotropical bird (Xenops minutus) reveals divergence, population expansion, and gene flow. Molecular Phylogenetics and Evolution 83:305-316. restriction site associated DNA sequencing for phylogeography. arXiv:1312.6439.

Hudson RR, Slatkin M, Maddison WP. 1992. Estimation of levels of gene flow from DNA sequence data. Genetics 132:583-589.

Hillier WH, Miller W, Birney E, Warren W, Hardison RC, Ponting CP, Bork P, Burt DW, Groenen MAM, Delaney ME, Dodgson JB, Chinwalla AT, Cliften PF, Clifton SW, Delehaunty KD, Fronick C, Fulton RS, Graves TA, Kremitzi C, Layman D, Magrini V, McPherson JD, Miner TL, Minx P, Nash WE, Nhan MN, Nelson JO, Oddy LG, Pohl CS, Randall-Maher J, Smith SM, Wallis JW, Yang S-P, Romanov MN, Rondelli CM, Paton B, Smith J, Morrice D, Daniels L, Tempest HG, Robertson L, Masabanda JS, Griffin DK, Vignal A, Fillon V, Jacobbson L, Kerje S, Andersson L, Crooijmans RPM, Aerts J, van der Poel JJ, Ellegren H, Caldwell RB, Hubbard SJ, Grafham DV, Kierzek AM, McLaren SR, Overton IM, Arakawa H, Beattie KJ, Bezzubov Y, Boardman PE, Bonfield JK, Croning MDR, Davies RM, Francis MD, Humphray SJ, Scott CE, Taylor RG, Tickle C, Brown WRA, Rogers J, Buerstedde J-M, Wilson SA, Stubbs L, 
Ovcharenko I, Gordon L, Lucas S, Miller MM, Inoko H, Shiina T, Kaufman J, Salomonsen J, Skjoedt K, Wong GK-A, Wang J, Lio B, Wang J, Yu J, Yang H, Nefedov M, Koriabine M, deJong PJ, Goodstadt L, Webber C, Dickens NJ, Letunic I, Suyama M, Torrents D, von Mering C, Zdobnov EM, Makova K, Nekrutenko A, Elnitski L, Eswara P, King DC, Yang S, Tyekucheva S, Radakrishnan A, Harris RS, Chiaromonte F, Taylor J, He J, Rijnkels M, Griffiths-Jones S, Ureta-Vidal A, Hoffman MM, Severin J, Searle SMJ, Law AS, Speed D, AG, Paterson AH, Ivarie R, Brandstrom M, Axelsson E, Backstrom N, Berlin S, Webster MT, Pourquie O, Reymond A, Ucla C, Antonarakis SE, Long M, Emerson JJ, Betrán E, Dupanloup I, Kaessmann H, Hinrichs AS, Bejerano G, Furey TS, Harte RA, Raney B, Siepel A, Kent WJ, Haussler D, Eyras E, Castelo R, Abril JF, Castellano S, Camara F, Parra G, Guigo R, Bourque G, Tesler G, Pevzner PA, Smit A, Fulton LA, Mardis ER, Wilson RK. 2004. Sequence and comparative analysis of the chicken genome provide unique perspectives on vertebrate evolution. Nature, 432, 695-716.

461 Ilut DC, Nydam ML, Hare MP. 2014. Defining loci in restriction-based reduced representation genomic data from nonmodel species: sources of bias and diagnostics for optimal clustering. BioMed Research International 2014:675158. 
Leaché AD, Harris RB, Maliska ME, Linkem CW. 2013. Comparative species divergence across eight triplets of spiny lizards (Sceloporus) using genomic sequence data. Genome Biology and Evolution 5:2410-2419.

Lewontin RC. 1974. The genetic basis of evolutionary change. New York: Columbia University Press.

Lu F, Lipka AE, Glaubitz J, Elshire R, Cherney JH, Casler MD, Buckler ES, Costich DE. 2013. Switchgrass genomic diversity, ploidy, and evolution: novel insights from a network-based SNP discovery protocol. PLoS Genetics 9:e1003215.

Lunter G, Goodson M. 2011. Stampy: a statistical algorithm for sensitive and fast mapping of Illumina sequence reads. Genome Research 21:936-939.

Maley JM. 2012. Ecological speciation of King Rails (Rallus elegans) and Clapper Rails (Rallus longirostris). D. Phil. Dissertation, Louisiana State University.

Maley JM, Brumfield RT. 2013. Mitochondrial and next-generation sequence data used to infer phylogenetic relationships and species limits in the Clapper/King rail complex. The Condor 115:316-329.

Mastretta-Yanes A, Arrigo N, Alvarez N, Jorgensen TH, Pinero D, Emerson BC. 2015. Restriction site-associated DNA sequencing, genotyping error estimation and de novo assembly optimization for population genetic inference. Molecular Ecology Resources 15:28-41. 
493 Nybom H. 2004. Comparison of different nuclear DNA markers for estimating intraspecific genetic 494 diversity in plants. Molecular Ecology 13:1143-1155.

495

496 Nylander JAA. 2004. MrAIC.pl. Available at: http://www.abc.se/ nylander (Accessed 12/20/2013) 497

498 Paradis E, Claude J, Strimmer K. 2004. APE: analyses of phylogenetics and evolution in R language. 499 Bioinformatics 20:289-290.

500

501 Parchman TL, Gompert Z, Mudge J, Schilkey FD, Benkman CW, Buerkle CA. 2012. Genome-wide 502 association genetics of an adaptive trait in lodgepole pine. Molecular Ecology 21:2991-3005.

503

504 Peterson BK, Weber JN, Kay EH, Fisher HS, Hoekstra HE. 2012. Double digest RADseq: an 505 inexpensive method for de novo SNP discovery and genotyping in model and non-model $506 \quad$ species. PloS One 7:e37135.

507

508 Pop M, Salzberg SL. 2008. Bioinformatics challenges of new sequencing technologies. Trends in $509 \quad$ Genetics, 24:142-149.

510

511 Python Software Foundation. 2007. Python version 2.7. Available at: http://www.python.org (Accessed $512 \quad 12 / 20 / 2013)$.

513

514 R Core Team. 2014. R: A language and environment for statistical computing. Available at:

$515 \quad$ http://www.R-project.org/ (Accessed 12/20/2013)

516 
517 Reitzel AM, Herrera S, Layden MJ, Martindale MQ, Shank TM. 2013. Going where traditional markers have not gone before: Utility of an promise for RAD sequencing in marine invertebrate phylogeography and population genomics. Molecular Ecology 22:2953-2970.

521 Remsen, JV Jr. 2003. Family Furnariidae (Ovenbirds). In: del Hoyo J, et al., eds. Handbook of the 522 Birds of the World. Barcelona: Lynx Edicions, 162-357. Faivre N, Loire E, Lourenco JM, Nabholz B, Roux C, Tsagkogeorga G, Weber AAT, Weinert LA, Belkhir K, Bierne N, Glémin S, Galtier N. 2014. Comparative population genomics in animals uncovers the determinants of genetic diversity. Nature 515:261-263. Bioinformatics 19:1572-1574.

535 Smith BT, Harvey MG, Faircloth BC, Glenn TC, Brumfield RT. 2013. Target capture and massively parallel sequencing of ultraconserved elements (UCEs) for comparative studies at shallow evolutionary time scales. Systematic Biology 63:83-95. 
539 Smith BT, McCormack JE, Cuervo AM, Hickerson MJ, Aleixo A, Cadena CD, Pérez Eman JE, Burney CW, Xie X, Harvey MG, Faircloth BC, Glenn TC, Derryberry EP, Prejean J, Fields S, Brumfield RT. 2014. The drivers of tropical speciation. Nature, 515, 406-409.

Sovic MG, Fries AC, Lisle Gibbs H. In press. AftrRAD: A pipeline for accurate and efficient de novo assembly of RADseq data. Molecular Ecology Resources doi:10.1111/1755-0998.12378. postglacial colonization routes in Europe. Molecular Ecology, 7, 453-464.

Thornton K. 2003. libsequence: a C++ class library for evolutionary genetic analysis. Bioinformatics 19: $2325-2327$.

Trapnell C, Salzberg SL. 2010. How to map billions of short reads onto genomes. Nature Biotechnology 27:455-457. of the National Academy of Sciences 107:9264-9269. 
562 Zhang RM, Zhang DX, Zhu T, Yang Z. 2011. Evaluation of a Bayesian coalescent method of species 563 delimitation. Systematic Biology 60:747-761.

564 


\section{Figure 1 (on next page)}

Figure 1: Two ways in which similarity thresholds can result in spurious assemblies.

(a) over-splitting occurs when reads from different alleles from the same genomic position are spuriously split into multiple loci due to lower similarity than the similarity threshold parameter, and (b) under-splitting occurs when reads from different genomic positions are clustered into a single locus due to higher similarity than the similarity threshold parameter. Gray bars represent identical sequence across reads, whereas colored squares represent alternate alleles at SNPs. 
a)

\section{Over-splitting}

\section{genome}

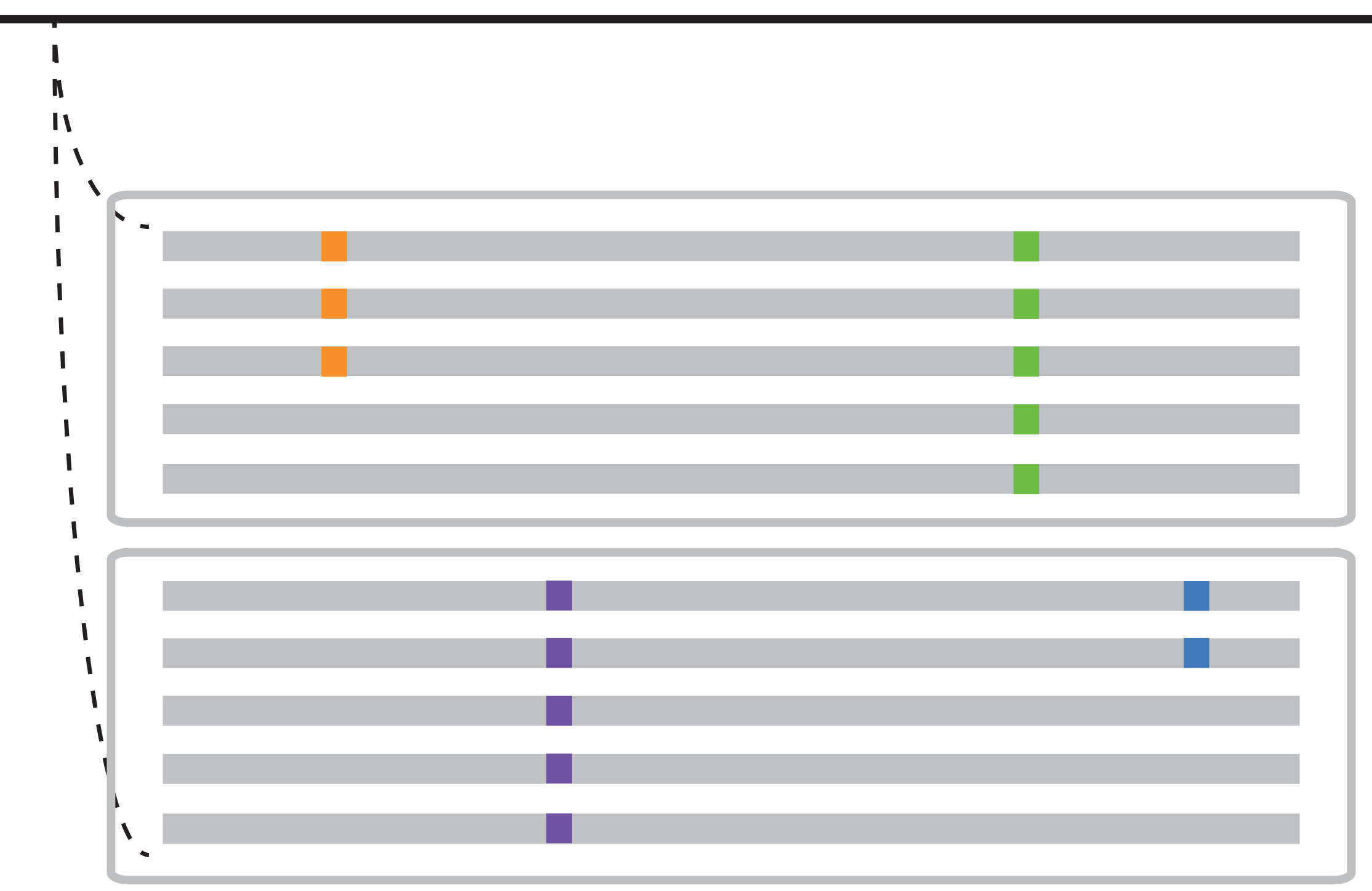

b)

Under-splitting

Assembly locus 1

Assembly locus 2

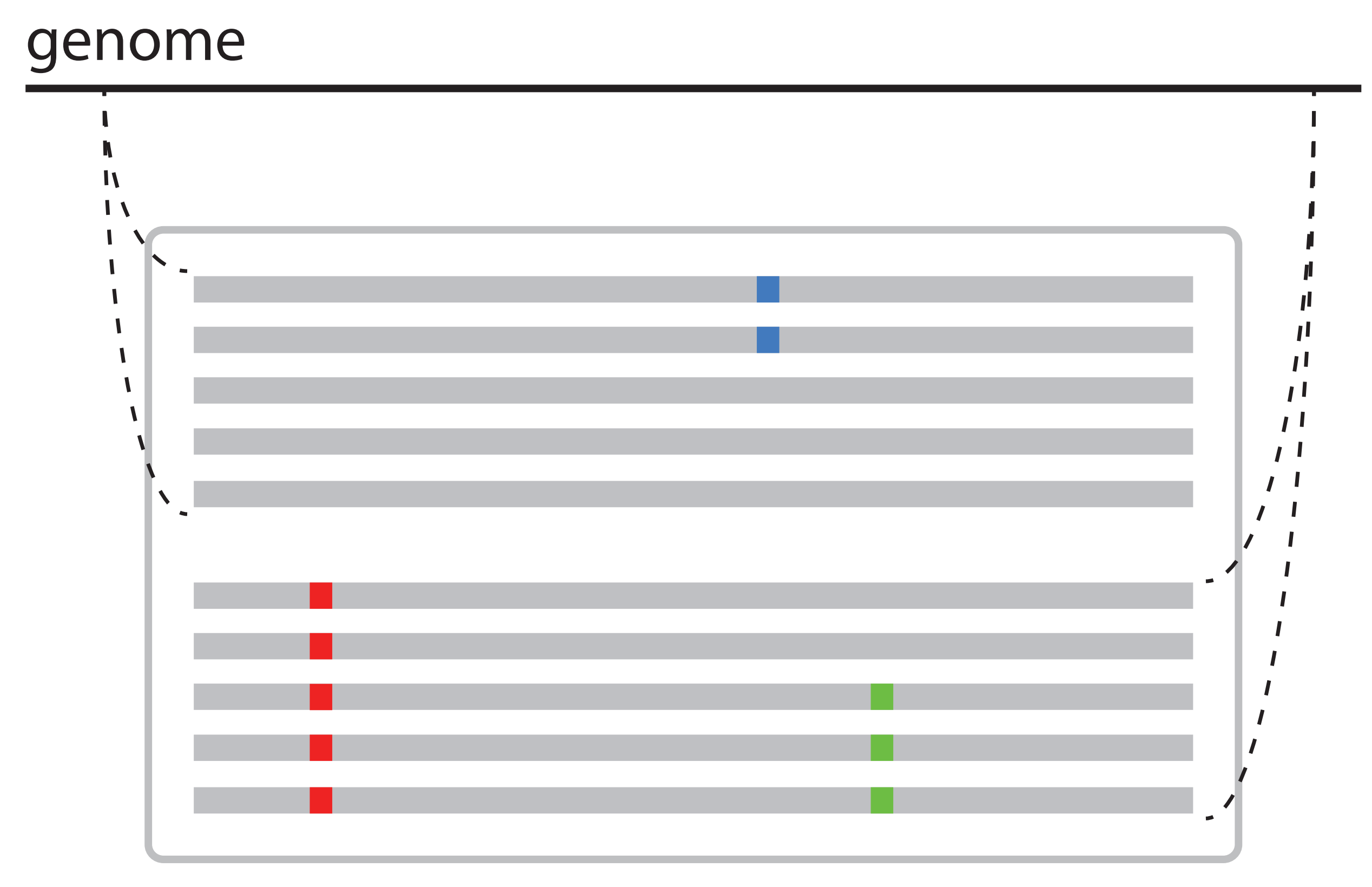

One locus in assembly 


\section{Table $\mathbf{1}$ (on next page)}

Table 1: Attributes and summary statistics (standard deviation across loci) of datasets assembled under the similarity thresholds examined. 


\begin{tabular}{|c|c|c|c|c|}
\hline & Threshold & Loci & $\begin{array}{l}\text { Individuals } \\
\text { Represented } \\
\text { per Locus }\end{array}$ & $\begin{array}{l}\text { Segregating } \\
\text { Sites per } \\
\text { Locus }\end{array}$ \\
\hline \multirow{7}{*}{ 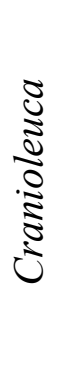 } & 99 & 147123 & $4.14(1.82)$ & $0.20(0.44)$ \\
\hline & 98 & 145423 & $4.2(1.82)$ & $0.30(0.63)$ \\
\hline & 97 & 144475 & $4.21(1.82)$ & $0.34(0.74)$ \\
\hline & 96 & 143780 & $4.22(1.82)$ & $0.38(0.86)$ \\
\hline & 95 & 142897 & $4.23(1.82)$ & $0.41(0.98)$ \\
\hline & 94 & 141880 & $4.23(1.82)$ & $0.44(1.11)$ \\
\hline & 93 & 140801 & $4.24(1.81)$ & $0.48(1.26)$ \\
\hline \multirow{7}{*}{ 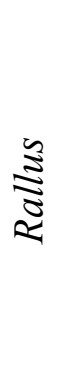 } & 99 & 100086 & $3.3(1.31)$ & $0.17(0.41)$ \\
\hline & 98 & 99300 & $3.31(1.31)$ & $0.24(0.60)$ \\
\hline & 97 & 98680 & $3.31(1.31)$ & $0.28(0.73)$ \\
\hline & 96 & 98206 & $3.31(1.3)$ & $0.30(0.83)$ \\
\hline & 95 & 97808 & $3.31(1.3)$ & $0.33(0.93)$ \\
\hline & 94 & 97321 & $3.31(1.3)$ & $0.36(1.07)$ \\
\hline & 93 & 96776 & $3.32(1.3)$ & $0.40(1.22)$ \\
\hline \multirow{7}{*}{ 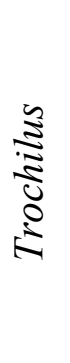 } & 99 & 125594 & $3.83(1.67)$ & $0.32(0.56)$ \\
\hline & 98 & 125966 & 3.87 (1.7) & $0.46(0.77)$ \\
\hline & 97 & 125697 & $3.88(1.7)$ & $0.51(0.87)$ \\
\hline & 96 & 125437 & $3.88(1.7)$ & $0.54(0.95)$ \\
\hline & 95 & 125118 & 3.89 (1.7) & $0.56(1.02)$ \\
\hline & 94 & 124669 & $3.89(1.7)$ & $0.59(1.13)$ \\
\hline & 93 & 123926 & $3.9(1.7)$ & $0.62(1.25)$ \\
\hline \multirow{7}{*}{$\frac{2}{2}$} & 99 & 155933 & $3.77(1.71)$ & $0.65(0.79)$ \\
\hline & 98 & 158496 & $3.94(1.74)$ & $1.05(1.17)$ \\
\hline & 97 & 158281 & $4(1.74)$ & $1.25(1.41)$ \\
\hline & 96 & 158328 & $4.01(1.74)$ & $1.35(1.56)$ \\
\hline & 95 & 158078 & $4.02(1.74)$ & $1.40(1.66)$ \\
\hline & 94 & 157534 & $4.02(1.74)$ & $1.45(1.76)$ \\
\hline & 93 & 156640 & $4.03(1.74)$ & $1.50(1.87)$ \\
\hline
\end{tabular}


Figure 2 (on next page)

Figure 2: The impact of similarity thresholds on empirical datasets from four bird lineages.

(a) Stringent similarity thresholds resulted in fewer unique alleles per locus relative to more liberal thresholds. (b) Putative over-split loci (connected by dashed lines) were more frequent in datasets assembled at stringent similarity thresholds, whereas loci containing under-split reads (solid lines) occurred at low frequency across all similarity thresholds (lines are overlapping). 
a)

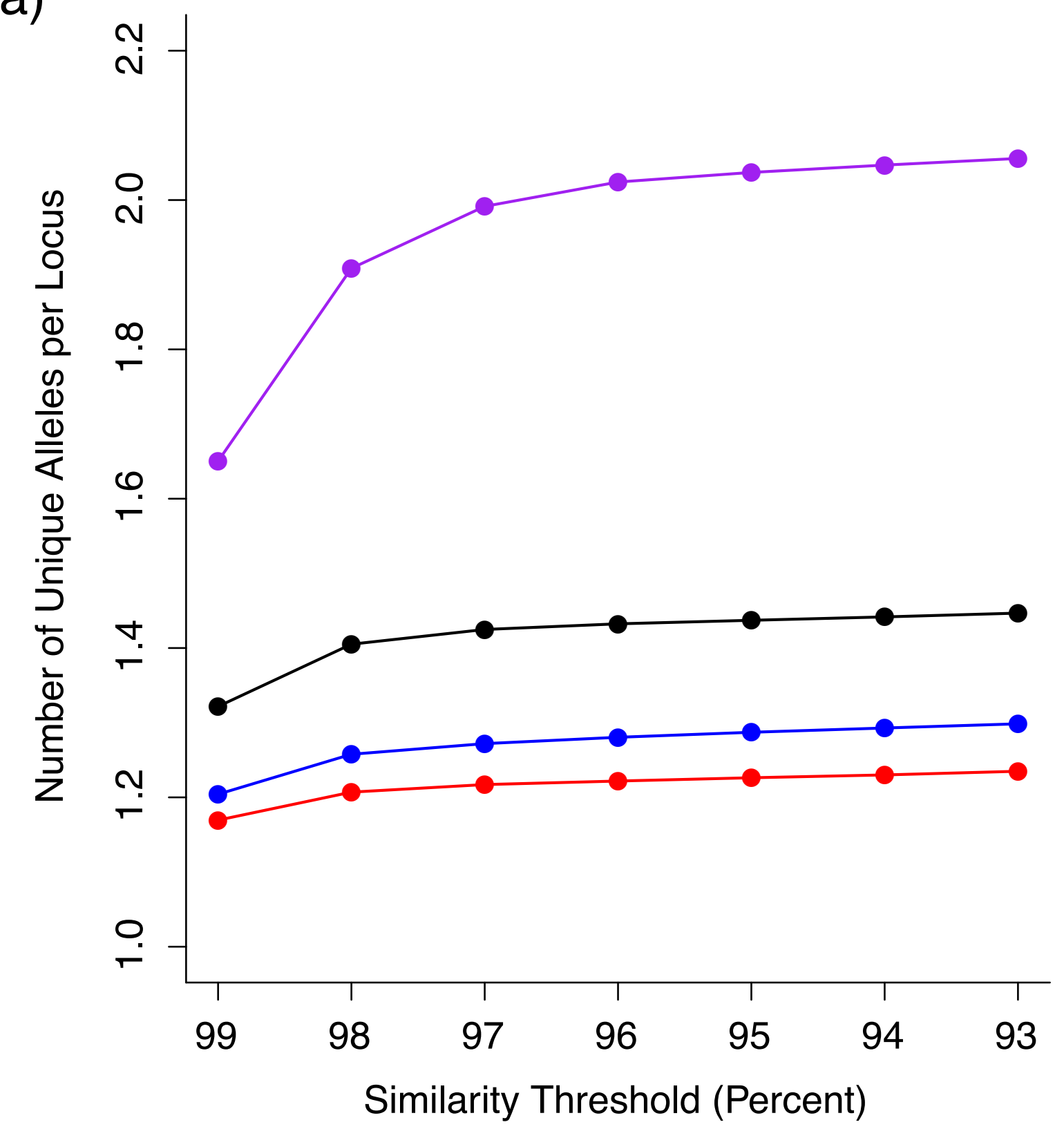

b)

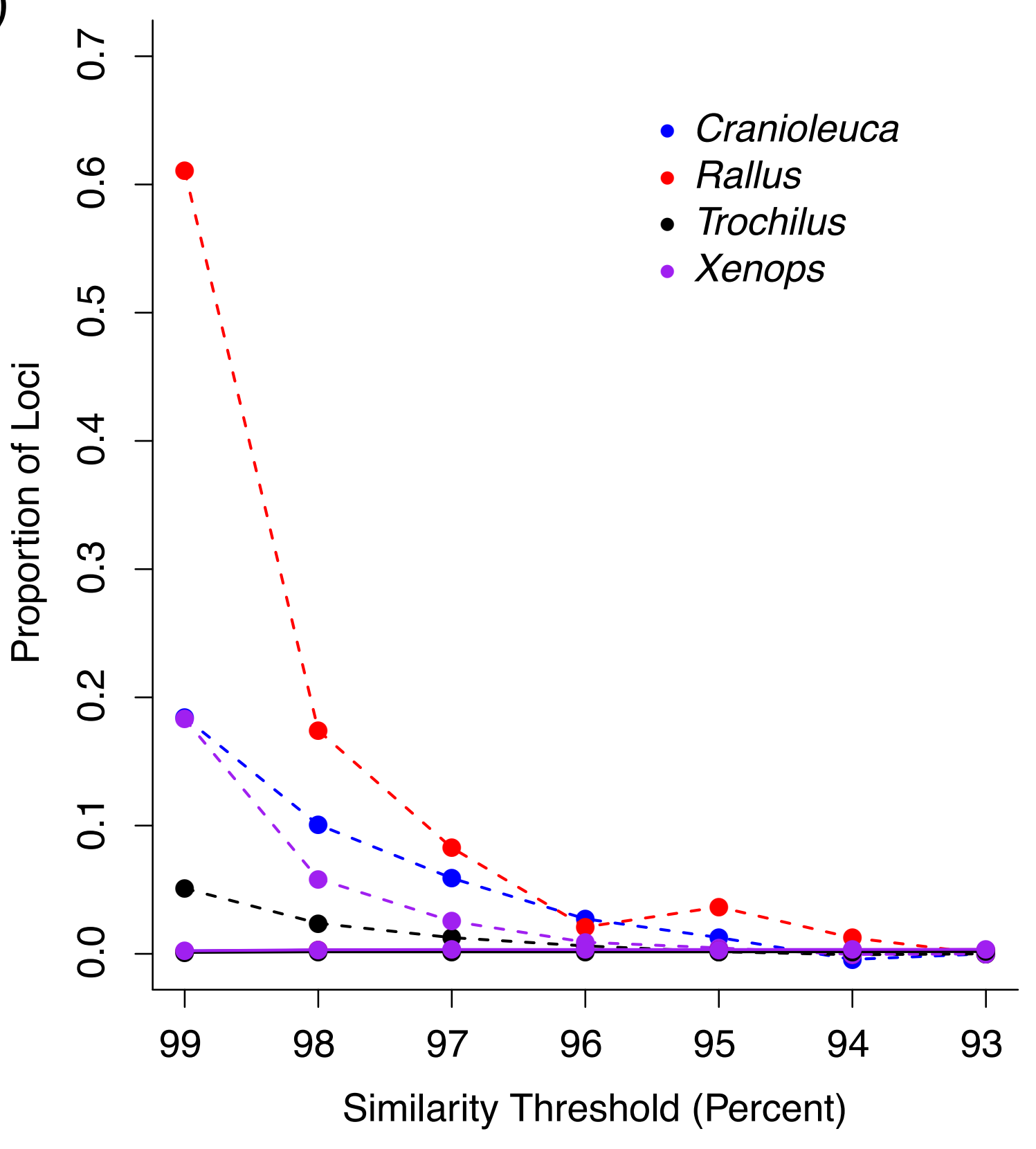


Figure $\mathbf{3}$ (on next page)

Figure 3: The impact of similarity thresholds on population genetic parameter estimates

The similarity threshold applied impacts (a) mean pairwise Jukes-Cantor corrected genetic distance between individuals, (b) mean $F_{S T}$ between populations, (c) mean gene tree depth and (d) ancestral theta based on a coalescent model. 
a)

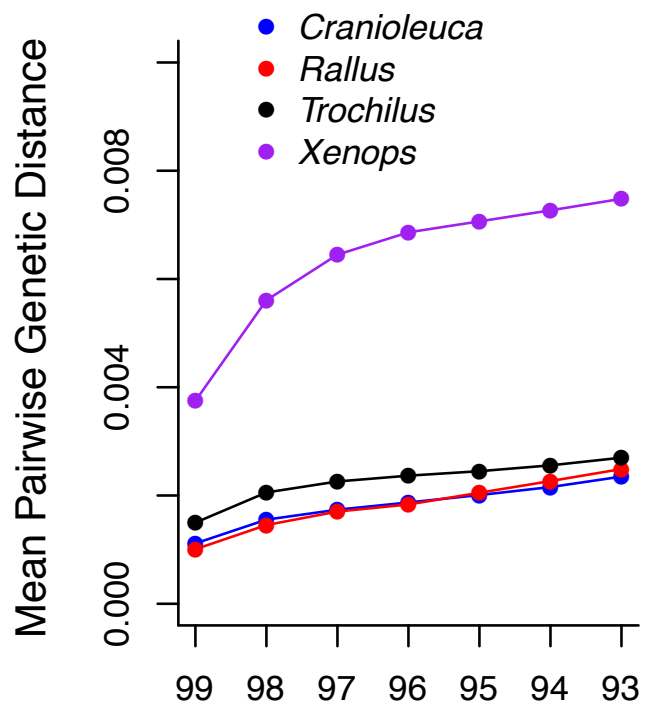

Similarity Threshold (Percent)

c)

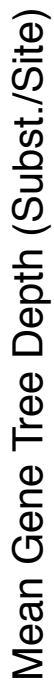

b)

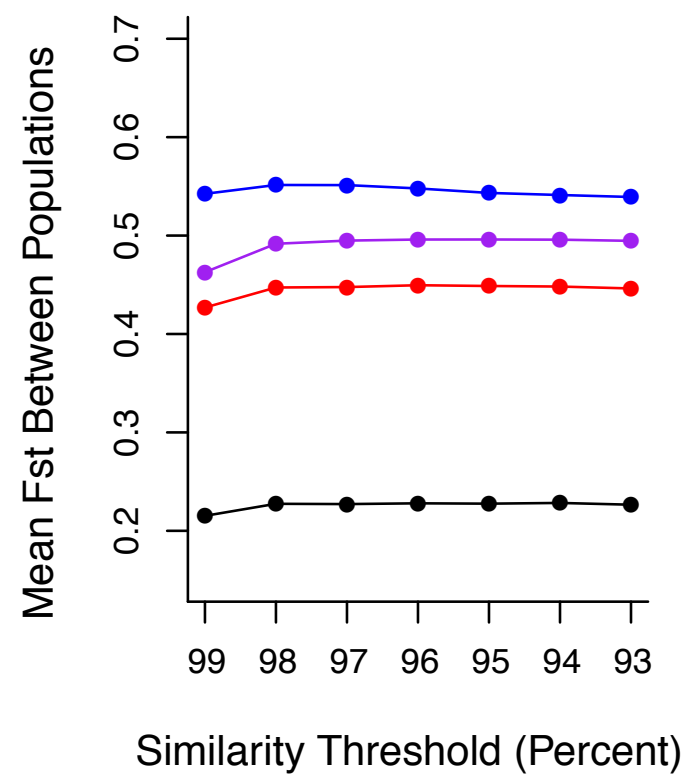

d)

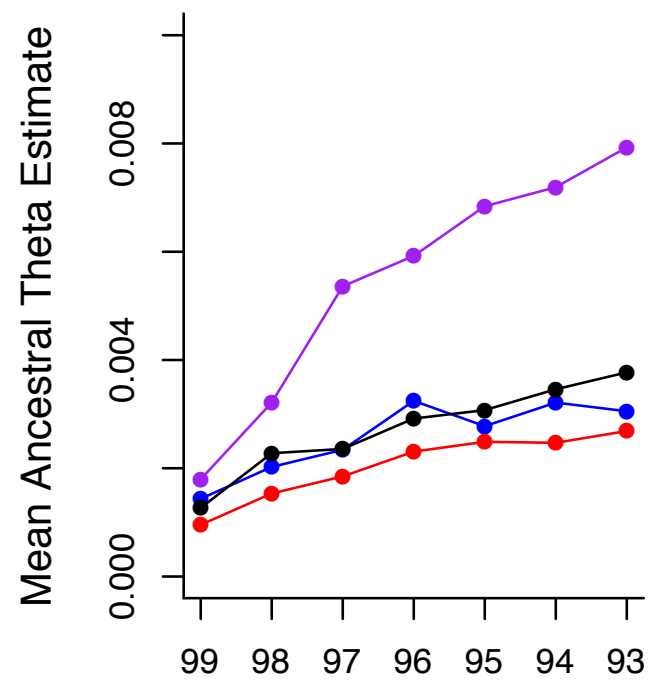

Similarity Threshold (Percent) 